\title{
Validation of separated source frequency delivery for a fiber-coupled heterodyne displacement interferometer
}

\author{
Arjan J. H. Meskers, ${ }^{*}$ Jo W. Spronck, and Robert H. Munnig Schmidt \\ Delft University of Technology, Department of Precision and Microsystems Engineering, Mekelweg 2, 2628 CD, Delft, The Netherlands \\ *Corresponding author: a.j.h.meskers@tudelft.nl
}

Received April 29, 2014; revised June 27, 2014; accepted June 30, 2014; posted July 1, 2014 (Doc. ID 211130); published July 31, 2014

\begin{abstract}
The use of optical fibers presents several advantages with respect to free-space optical transport regarding sourcefrequency delivery to individual heterodyne interferometers. Unfortunately, fiber delivery to individual coaxial heterodyne interferometers leads to an increase of (periodic) nonlinearity in the measurement, because transporting coaxial frequencies through one optical fiber leads to frequency mixing. Coaxial beams thus require delivery via free-space transportation methods. In contrast, the heterodyne interferometer concept discussed in this Letter is based on separated source frequencies, which allow for fiber delivery without additional nonlinearity. This investigation analyzes the influence of external disturbances acting on the two fibers during delivery, causing asymmetry in phase between the two fibers (first-order effect), and irradiance fluctuations (second-order effect). Experiments using electro-optic phase modulation and acousto-optic irradiance modulation confirmed that the interferometerconcept can measure with sub-nanometer uncertainty using fiber delivered source frequencies, enabling fully fiber-coupled heterodyne displacement interferometers. (c) 2014 Optical Society of America

OCIS codes: (120.0120) Instrumentation, measurement, and metrology; (120.3180) Interferometry; (120.3940)

Metrology; (120.4570) Optical design of instruments; (120.4820) Optical systems; (120.5050) Phase measurement.

http://dx.doi.org/10.1364/OL.39.004603
\end{abstract}

Optical interferometry is a widely applied method for measuring displacement in the fields of metrology and precision engineering. It is used for tracking precision stages [1] in lithography systems in the semiconductor industry, space-based gravitational wave detection [2], and in atomic force microscopy [3]. Additionally, it is used as a means for calibrating other measurement tools [ $\underline{4}]$.

There are many error sources that affect the measurement uncertainty of a heterodyne displacement interferometer, especially when aiming for sub-nanometer uncertainty over a large measurement range (i.e., optical measurement pathways of several meters). The impact of several of these error sources, e.g., thermal influence and refractive index fluctuations, can be reduced by external placement of the heat sources (laser source and electronic readout) or operating in vacuum. However, some error sources remain, one of which is periodic nonlinearity (PNL). This error source is attributed by ghost reflections, by imperfections in polarization alignment, and by the polarization quality of both the source frequencies and polarizing optics [5-9].

In an effort to meet the industrial need for system layout flexibility and robustness, optical fibers are being employed, replacing free-space optical pathways [see Figs. 1(a) and 1(b)]. The replacement of free-space optical pathways is driven by their inflexible layout and their (high) sensitivity to environmental disturbances. Still, it is difficult for coaxial based interferometer systems to deliver the source frequencies directly to individual interferometers within a host system. Research [10] indicates that fiber delivery of a coaxial beam with one polarization maintaining single-mode fiber is possible, but increases PNL due to frequency mixing within the optical fiber. This necessitates separated source frequency transport using two optical fibers, and coaxially recombining them into a free-space beam inside the host system [see Fig. 1(b)]. The flexibility of optical fibers allows the externally placed equipment to be easily relocated, as seen in current commercial systems from Agilent Technologies.

The two source frequencies are ideally combined into a coaxial beam at the interferometer creating a fully fiber-coupled instrument; unfortunately, this is costly and highly impractical with regard to space consumption and complexity. The solution is to combine the source frequencies into a coaxial beam only once (at the remote optical combiner) and branch off parts of the free-space beam to individual interferometers [see Fig. 1(b)].

Frequency leakage (and thereby PNL) can be prevented by spatially separating the source frequencies throughout the interferometer system until detection [11-18]. The main advantage of separated source frequency delivery is that it can take place either free-space as well as via optical fibers, offering a reconfigurable means of optical transport at minimum space consumption and enabling complete fiber-coupled heterodyne displacement interferometers [see Fig. 1(c)].

The research discussed in this Letter is based upon previous research on the "Delft interferometer" [1416], which reported on its measurement performance when fed with two fiber-delivered source frequencies (using polarization maintaining single-mode fibers) [15]. It did not, however, extensively analyze time variant influences of external disturbances acting on the fiber delivery. The disturbances influencing the optical fibers were not predetermined or repeatable, two important prerequisites for validation of the concept's ability to reliably operate with fiber delivered source frequencies. Non-symmetric external disturbances affect the two individual optical fibers differently, leading to timevariant phase differences between the two source 

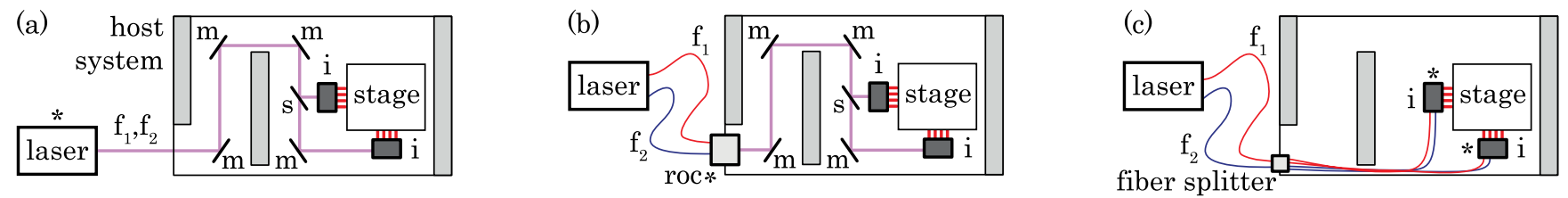

Fig. 1. (a) In a free-space system, a coaxial beam (purple) is delivered to interferometers (i) inside a host system to measure stage displacement, using mirrors (m), and a beam splitter (s). (b) A more practical commercial system layout as is found in current lithography machines uses optical-fiber delivery, in which the two source frequencies are coaxially combined by a remote optical combiner (roc), resulting in a free-space beam. (c) The most practical system consists of fully fiber-coupled interferometers, easing system integration. An asterisk denotes the generation of a reference signal. Note that transport of interference signals from interferometers is less stringent and has already proven itself using optical fibers and external readout (not shown).

frequencies. These differences can be in the order of several hundred degrees [15] and potentially affect the measurement uncertainty.

In this Letter, the Delft interferometer's ability to operate fiber-coupled will be further investigated, as part of achieving an overall characterization of the interferometer concept. The final aim is to develop a modular heterodyne displacement interferometer that measures with sub-nanometer uncertainty while using optical fibers for the optical transport of both the source frequencies and interference signals.

The experimental setup illustrated in Fig. 2 uses a twomode helium-neon gas laser (Thorlabs HRS015), and two acousto-optic modulators (aom), $\left(\right.$ aom $_{1,2}$ ISOMET OAM1141-T40-2 and drivers 531C-L, operating at 39 and $41 \mathrm{MHz}$ ) generate a $2 \mathrm{MHz}$ split frequency. The applied electro-optic modulator (eom) (eom Thorlabs EO-phase modulator EO-PM-NR-C1) modulates the phase of one of the two source frequencies (simulating air pressure fluctuations, temperature fluctuations, air turbulence, and fiber vibrations) resulting in a time varying phase difference between $f_{1}$ and $f_{2}$. Furthermore, standard optical components have been applied from Thorlabs, and a fiber coupled phase-measurement board from Agilent Technologies (N1225A). The setup uses free-space beam delivery in a normal air environment but could be equipped with optical fibers as well. The experiment contains two interferometers, one "classical" interferometer (using $\mathrm{PD}_{1,2}$ ), and one Delft interferometer (using $\mathrm{PD}_{3,4}$ ). The classical interferometer interprets the eom's phase modulation as a displacement, while the Delft interferometer excludes this disturbance, which is analyzed.

Two linearly (vertically) polarized beams are delivered to the two interferometers,

$$
\begin{aligned}
& J_{1}=E_{1} \cdot\left[\begin{array}{l}
0 \\
1
\end{array}\right] e^{i\left(\omega_{1} t+\theta\right),} \\
& J_{2}=E_{2} \cdot\left[\begin{array}{l}
0 \\
1
\end{array}\right] e^{i\left(\omega_{2} t+\varphi\right),}
\end{aligned}
$$

where $\omega_{1,2}=2 \pi f_{1,2}\left(\right.$ with $\left.f_{1}<f_{2}\right)$ and $\theta$ and $\varphi$ represent (unknown and uncontrollable) time varying phase changes in $f_{1}$ and $f_{2}$, respectively, caused by the measurement environment. Using rhomboids (rb) for beam overlap in the classical interferometer prevents frequency leakage of $f_{1}$ into $f_{2}$. Having both beams vertically polarized removes the need for an analyzer at the PDs. At $\mathrm{PD}_{1}$ the obtained interference signal is

$$
\begin{aligned}
& I_{\mathrm{PD}_{1}} \propto J_{1}^{\dagger} \cdot J_{2}, \\
& I_{\mathrm{PD}_{1}} \propto E_{1} E_{2} e^{i\left(\left\{\omega_{2}-\omega_{1}\right\} t+\varphi-\theta\right)} .
\end{aligned}
$$

Equation (4) shows the reference frequency for the classical interferometer consisting of the frequency difference between $f_{1}$ and $f_{2}$ (i.e., $\omega_{2}-\omega_{1}$ ).

The eom modulates the phase of $f_{2}$ according to

$$
\varphi_{\mathrm{eom}}=A \cos \left(\omega_{\mathrm{eom}} t\right)
$$

where $\omega_{\text {eom }}=2 \pi f_{\text {eom }}$ describes the linear electric field that is applied to the eom's electro-optic crystal, which gives rise to an electric field dependent birefringence.

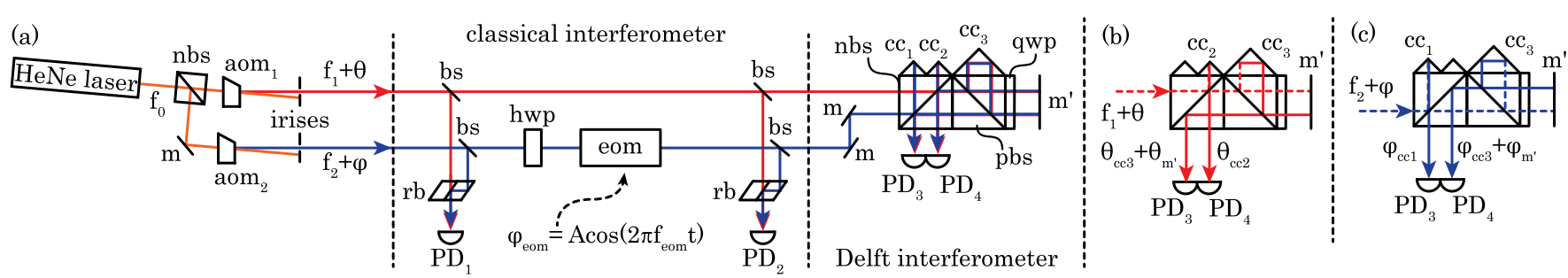

Fig. 2. (a) Schematic of the experimental setup where an eom introduces a relative phase difference $\varphi_{\text {eom }}$, between $f_{1}$ and $f_{2}$. The half wave plate (hwp) in front of the eom is used for adjusting the polarization alignment between $f_{2}$ and the eom crystal. (b), (c) Optical pathways of respectively $f_{1}$ and $f_{2}$ through the interferometer (dotted lines denote beams in the lower plane whereas solid lines are beams in the upper plane, see also $[15,16])$. Note that the tested interferometer was not a monolithic structure as

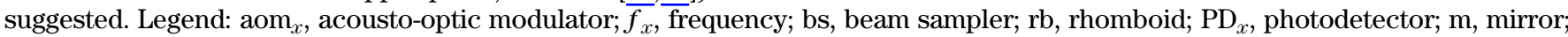
nbs neutral beam splitter, pbs, polarizing beam splitter; $\mathrm{cc}_{x}$, cube corner reflector; qwp, quarter wave plate; and $\mathrm{m}^{\prime}$, target mirror. 
Analysis for the other PDs according to Fig. 2 leads to

$$
\begin{gathered}
I_{\mathrm{PD}_{2}} \propto E_{1} E_{2} e^{i\left(\left\{\omega_{2}-\omega_{1}\right\} t+\varphi+\varphi_{\mathrm{eom}}-\theta\right)}, \\
I_{\mathrm{PD}_{3}} \propto E_{1} E_{2} e^{i\left(\left\{\omega_{2}-\omega_{1}\right\} t+\varphi+\varphi_{\mathrm{eom}}+\varphi_{\mathrm{cc} 1}-\theta-\theta_{\mathrm{cc} 3}-\theta_{m^{\prime}}\right)} \\
I_{\mathrm{PD}_{4}} \propto E_{1} E_{2} e^{i\left(\left\{\omega_{2}-\omega_{1}\right\} t+\varphi+\varphi_{\mathrm{eom}}+\varphi_{\mathrm{cc} 3}+\varphi_{m^{\prime}}-\theta-\theta_{\mathrm{cc} 2}\right)} .
\end{gathered}
$$

Since the interferometer, illustrated in Fig. 2, was not monolithic, vibrations of individual components have been taken into account explaining the many additional phase terms. The signs of $\theta_{m^{\prime}}$ and $\varphi_{m^{\prime}}$ are opposite in Eqs. (7) and (8), which is caused by an opposite shift of the beat frequency at the two detectors. To clarify, when target $m^{\prime}$ displaces toward the interferometer, $\mathrm{PD}_{3}$ encounters a decrease of the beat frequency, $f_{\text {beat PD3 }}=f_{2}-\left(f_{1}+\theta_{m^{\prime}}\right)$, whereas $\mathrm{PD}_{4}$ encounters an increase of the beat frequency, $f_{\text {beat PD4 }}=\left(f_{2}+\varphi_{m^{\prime}}\right)-f_{1}$.

The classical interferometer will interpret $\varphi_{\text {eom }}$ as an apparent displacement, obtained through a differential operation between $\mathrm{PD}_{1}$ and $\mathrm{PD}_{2}$ :

$$
I_{\text {classical }} \propto E_{1} E_{2} e^{i\left[\begin{array}{c}
\left(\left\{\omega_{2}-\omega_{1}\right\} t+\varphi+\varphi_{\mathrm{eom}}-\theta\right)- \\
\left(\left\{\omega_{2}-\omega_{1}\right\} t+\varphi-\theta\right)
\end{array}\right],}
$$

$$
I_{\text {classical }} \propto E_{1} E_{2} e^{i\left(\varphi_{\text {eom }}\right)} .
$$

Equation (10) shows that the common noise terms $\theta$ and $\varphi$, and the frequencies $\omega_{1,2}$ cancel, resulting in the measurement of $\varphi_{\text {eom }}$. The same differential operation is performed for the Delft interferometer:
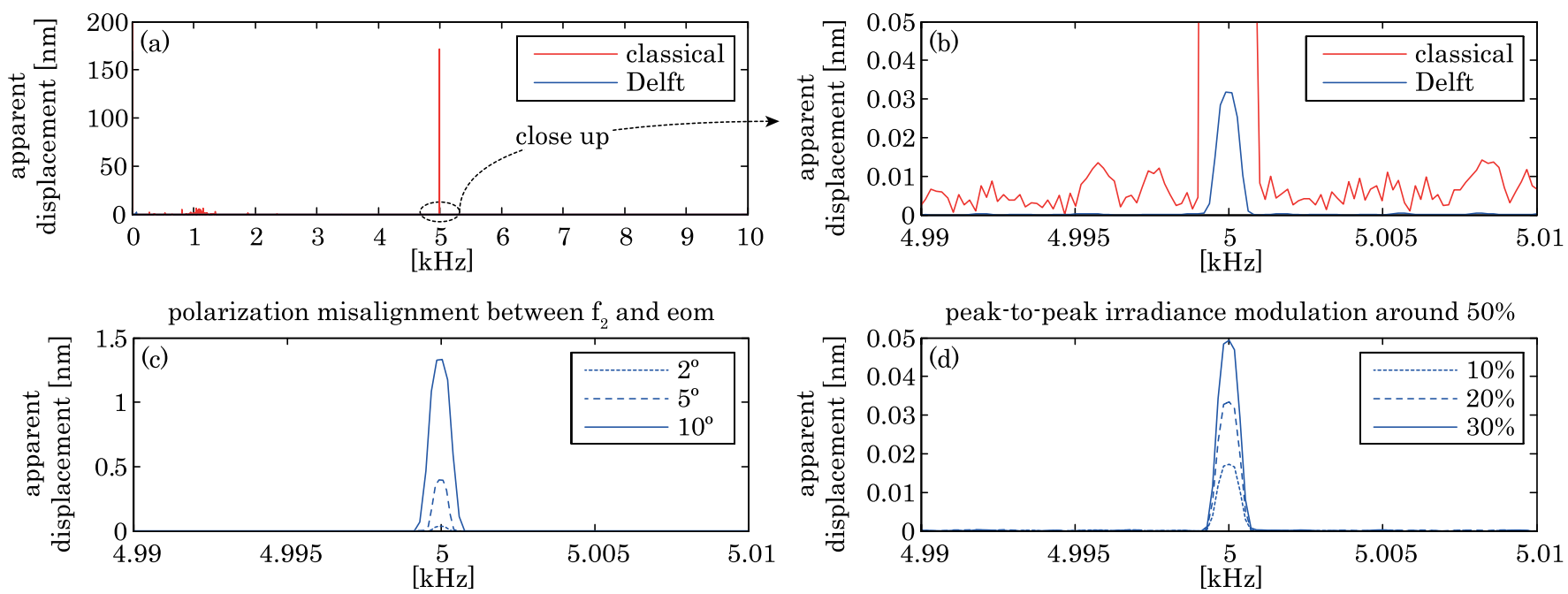

$I_{\text {Delft }}$

$$
\propto E_{1} E_{2} e^{i\left[\begin{array}{c}
\left(\left\{\omega_{2}-\omega_{1}\right\} t+\varphi+\varphi_{\mathrm{eom}}+\varphi_{\mathrm{cc} 3}+\varphi_{m^{\prime}}-\theta-\theta_{\mathrm{cc} 2}\right) \\
-\left(\left\{\omega_{2}-\omega_{1}\right\} t+\varphi+\varphi_{\mathrm{eom}}+\varphi_{\mathrm{cc} 1}-\theta-\theta_{\mathrm{cc} 3}-\theta_{m^{\prime}}\right)
\end{array}\right],}
$$

$I_{\text {Delft }} \propto E_{1} E_{2} e^{i\left(\varphi_{\mathrm{cc} 3}-\varphi_{\mathrm{cc} 1}-\theta_{\mathrm{cc} 2}+\theta_{\mathrm{cc} 3}+\left(\varphi_{m^{\prime}}+\theta_{m^{\prime}}\right)\right)}$.

The absence of $\varphi_{\text {eom }}$ in Eq. (12) shows that any phase difference between the two separately delivered source frequencies is mitigated. This is due to the interferometer's layout that delivers any fiber induced disturbance to both detectors; a differential operation between the detectors then cancels all common terms.

Note that the phase due to displacement $\varphi_{m^{\prime}}+\theta_{m^{\prime}}$ is present twice, indicating that this interferometer actually consists of two interferometers that both have an optical resolution of $\lambda / 4$, due to the differential readout. This results in a final displacement resolution of $\lambda / 8$.

The results in Eqs. (10) and (12) were verified using the setup shown in Fig. 2. Data from both interferometers was obtained simultaneously and was subsequently fast Fourier transformed (FFT). The combination of the eom operating at a fixed frequency and applying an FFT resulted in a noise level of less than a picometer for the Delft interferometer [see Figs. 3(b)-3(d)]. The eom applied a phase modulation at $5 \mathrm{kHz}$ with a modulation depth of $\sim 95^{\circ}$, leading to an apparent displacement of $\sim 170 \mathrm{~nm}$ for the classical interferometer. This is indicated by the red peak in Fig. 3(a).

The influence of the eom is present in the classical interferometer's output (red), whereas it is not in the output of the Delft interferometer (blue) [see Fig. 3(a)]. These results are in agreement with Eqs. (10) and (12) and confirm that the disturbance from the eom is cancelled by the differential action between $\mathrm{PD}_{3,4}$.

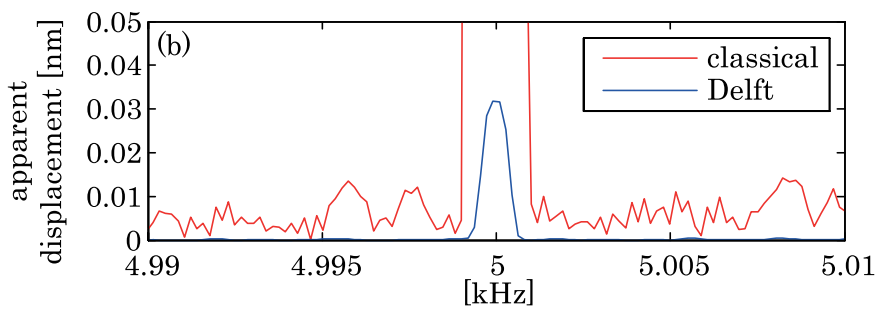

Fig. 3. (a) The eom has a phase modulation depth of $\sim 95^{\circ} \mathrm{nm}(170 \mathrm{~nm})$ at $f_{\text {eom }}=5 \mathrm{kHz}$, which is present in the output of the classical interferometer, $170 \mathrm{~nm}$ (red), while it is absent in the output of the Delft interferometer (blue). (b) Close-up of the result of (a), showing a residual error of $\sim 0.03 \mathrm{~nm}$. (c) The residual is affected by polarization misalignment between $f_{2}$ and the eom crystal, which causes both an additional interference signal and a modulation of the irradiance. (d) Influence of irradiance modulation was analyzed by amplitude modulation of aom $_{2}$ at $50 \%$ of the maximum irradiance $(\sim 15 \mu \mathrm{W}$ interference signal strength at detectors $\mathrm{PD}_{3,4}$ ), with the eom switched off and the hwp at the position as was used for generating the results illustrated in (a). 
However, a close up of Fig. 3(a), shown in Fig. 3(b), reveals a peak of $\sim 0.03 \mathrm{~nm}$ and indicates a phase disturbance suppression ratio of $\sim 5700(170 \mathrm{~nm} / 0.03 \mathrm{~nm})$, which suggests that the eom's phase disturbance is not completely mitigated. For further robustness improvement it is essential to understand where the residual originates from.

The error is the result of a differential operation between $\mathrm{PD}_{3,4}$ and can therefore only consist from signal differences between the two detectors. These differences could consist from a number of effects: the presence of additional interference signals, an unbalanced irradiance distribution between the detectors, or optical pathway asymmetry between the reference and measurement pathways (affecting the differential operation by a non-simultaneous arrival of a disturbance).

It is most likely that the residual consists of ghost reflections, which are a well-known source of additional interference signals. The target's first reflection can partially be transmitted by the polarizing beam splitter via polarization leakage, and could eventually be reinjected from the feeding direction (see Fig. 2). The amount of irradiance required for explaining the residual is already present even when using good antireflection coatings [19].

Another source of additional interference signals is formed by the eom. The eom consists of a birefringent crystal that is modulated over one axis, which makes it sensitive to polarization misalignment. When the linear polarization orientation of $f_{2}$ mismatches with the crystal's birefringent index, the beam is only partially phase modulated. This results in a primary interference signal at $5 \mathrm{kHz}$ between $f_{1}$ and $f_{2}$ [clearly visible with the classical interferometer in Fig. 3(a)] together with a secondary interference signal from $f_{2}$ itself, also at $5 \mathrm{kHz}$.

This hypothesis was verified by inducing polarization misalignment by rotation of a half-wave plate in front of the eom [Fig. 2(a)]. Increasing the polarization misalignment of $f_{2}$ resulted in a nonlinear increase of the error [see Fig. 3(c)]. Placing a Glan-Taylor polarizer behind the eom only resulted in a limited reduction of the effect, indicating that this error adds little to the residual.

Timing issues due to optical pathway asymmetry between the reference and the measurement pathway are present, but can be considered negligible in comparison to the influence from ghost reflections.

Additionally, polarization misalignment between $f_{2}$ and the eom crystal not only leads to an additional interference signal, it also results in an irradiance modulation at $5 \mathrm{kHz}$, due to the use of polarizing optics. When the eom was modulating phase using equal settings as used for generating the results illustrated in Figs. 3(a) and $3(\mathrm{~b})$, an irradiance modulation of less than $\overline{0.5 \%}$ was measured.

The influence of irradiance modulation of $f_{2}$ was further analyzed by modulating the amplitude of the driving signal of aom 2 , with the eom switched off [see Fig. 2(a)]. The irradiance was modulated up to $\pm 2.5 \mu \mathrm{W}$ around $\sim 7.5 \mu \mathrm{W}$ (AC-signal at detector), with a peak-to-peak modulation amplitude of $10 \%, 20 \%$, and $30 \%$. The results are illustrated in Fig. 3(d) and show a linear increase of the error as the modulation amplitude was enlarged.
The graphs illustrated in Figs. 3(c) and 3(d) show that the influence of the irradiance modulation on the residual error of $0.03 \mathrm{~nm}$, Fig. 3(b), is much smaller than the influence of polarization misalignment between $f_{2}$ and the eom's birefringent crystal.

It can be concluded that the residual of $0.03 \mathrm{~nm}$ consists of multiple effects. However, some of them will not be encountered during normal operation and some can be reduced. This holds for partial phase modulation by the eom when using single mode optical fibers and for ghost reflections and optical symmetry when employing monolithic optical structures, respectively.

The research in this Letter has demonstrated that the discussed interferometer can achieve subnanometer measurement uncertainty while using separated source frequency delivery. The interferometer can significantly mitigate time variant phase disturbances and irradiance fluctuations that result from external disturbances acting on source frequency transport.

These findings are an important step toward a PNL free heterodyne displacement interferometer that can measure with subnanometer uncertainty while being fully fiber coupled. For this instrument, the use of optical fibers leads to system modularity, which eases the integration into complex host systems. An additional advantage worth mentioning is that the pluggable nature of optical fibers potentially results in fast component replacement, which helps minimizing downtime of a host system upon component failure.

This work was supported by the Dutch IOP (IPT04001) in the Netherlands. The authors are thankful for the support by Agilent Technologies for providing equipment used during this research.

\section{References}

1. F. C. Demarest, Meas. Sci. Technol. 9, 1024C (1998).

2. T. Schuldt, M. Gohlke, D. Weise, U. Johann, A. Peters, and C. Braxmaier, Class. Quantum Grav. 26, 085008 (2009).

3. J. Schneir, T. McWaid, J. Alexander, and B. Wilfley, J. Vac. Sci. Technol. B 12, 3561 (1994).

4. P. de Groot, J. Biegen, J. Clark, X. Colonna de Lega, and D. Grigg, Appl. Opt. 41, 3853 (2002).

5. G. Fedotova, Meas. Sci. Technol. 23, 577 (1980).

6. R. Quenelle, Hewlett-Packard J. 34, 10 (1983).

7. C. Sutton, J. Phys. E 20, 1290 (1987).

8. W. Hou and G. Wilkening, Precis. Eng. 14, 91 (1992).

9. C. Wu and R. D. Deslattes, Appl. Opt. 37, 6696 (1998).

10. B. A. W. H. Knarren, S. J. A. G. Cosijns, H. Haitjema, and P. H. J. Schellekens, Precis. Eng. 29, 229 (2005).

11. M. Tanaka, T. Yamagami, and K. Nakayama, IEEE Trans. Instrum. Meas. 38, 552 (1989).

12. C.-M. Wu, J. Lawall, and R. D. Deslattes, Appl. Opt. 38, 4089 (1999).

13. T. Schmitz and J. Beckwith, J. Mod. Opt. 49, 2105 (2002).

14. K.-N. Joo, J. D. Ellis, J. W. Spronck, P. J. M. van Kan, and R. H. M. Schmidt, Opt. Lett. 34, 386 (2009).

15. J. D. Ellis, A. J. H. Meskers, J. W. Spronck, and R. H. M. Schmidt, Opt. Lett. 36, 3584 (2011).

16. A. J. H. Meskers, J. W. Spronck, and R. H. Munnig Schmidt, Opt. Lett. 39, 1949 (2014).

17. C. Weichert, P. Köchert, R. Köning, J. Flügge, B. Andreas, U. Kuetgens, and A. Yacoot, Meas. Sci. Technol. 23, 094005(2012).

18. J. Lawall and E. Kessler, Rev. Sci. Instrum. 71, 2669 (2000).

19. C. Wu, Opt. Commun. 215, 17 (2003). 